Cahiers de la recherche sur les droits

Cahiers

Fon les Droits fondamentaux

11 | 2013

Le droit de la famille en (r)évolutions

\title{
Famille et Convention européenne des droits de
} l'homme

Jean-Manuel Larralde

\section{(2) OpenEdition}

\section{Journals}

Édition électronique

URL : https://journals.openedition.org/crdf/4620

DOI : $10.4000 /$ crdf.4620

ISSN : 2264-1246

Éditeur

Presses universitaires de Caen

Édition imprimée

Date de publication : 1 novembre 2013

Pagination : 21-29

ISSN : 1634-8842

\section{Référence électronique}

Jean-Manuel Larralde, « Famille et Convention européenne des droits de l'homme », Cahiers de la

recherche sur les droits fondamentaux [En ligne], 11 | 2013, mis en ligne le 01 décembre 2014, consulté le 14 novembre 2022. URL : http://journals.openedition.org/crdf/4620 ; DOI : https://doi.org/10.4000/ crdf. 4620 


\title{
Famille et Convention européenne des droits de l'homme
}

\author{
Jean-Manuel LARRALDE \\ Professeur de droit public à l'Université de Caen Basse-Normandie \\ Centre de recherche sur les droits fondamentaux et les évolutions du droit (CRDFED, EA 2132)
}

I. L'effectivité de la notion de vie familiale

A. L'appréciation in concreto de la réalité familiale

B. La reconnaissance des effets spécifiques du lien marital

II. L'extension encadrée des droits liés à l'exercice de la vie familiale

A. La prise en compte de l'intérêt supérieur de l'enfant

B. La lutte contre les discriminations frappant les membres de la famille

Si les textes internationaux de protection des droits de l'homme sont souvent présentés comme des normes visant des sujets de droits individuels, cela ne veut pas dire pour autant qu'ils excluent toute idée de protection de certains groupes. Tel est le cas de la famille, explicitement prise en compte par plusieurs d'entre eux, avec une intensité variable. Dès la Déclaration universelle des droits de l'homme du 10 décembre 1948, on apprend ainsi qu'il existe un «droit à fonder une famille» (art. 16, $\left.\S_{1}\right)$ - qui renvoie notamment à la liberté nuptiale ${ }^{1}-$, et que cette famille constitue "l'élément naturel et fondamental de la société» qui «a droit à la protection de la société et de l'État " ${ }^{2}$. L'exigence d'une rémunération «équitable et satisfaisante» versée à chaque travailleur permet, enfin, d'assurer à sa famille « une existence conforme à la dignité humaine» $\left(\operatorname{art} .23, \S_{3}\right)^{3}$, puisque « toute personne a droit à un niveau de vie suffisant pour assurer sa santé, son bien-être et ceux de sa famille» (art. 25) ${ }^{4}$. À ces éléments s'ajoute l'interdiction posée par l'article $17, \$ 1$ du Pacte international relatif aux droits civils et politiques, qui prohibe les «immixtions arbitraires ou illégales» dans la famille. Certains textes sont même allés plus loin encore, en faisant de la famille un destinataire des devoirs de l'individu, qui doit notamment «préserver le développement harmonieux de la famille et œuvrer en faveur de la cohésion et du respect de cette famille» et a le devoir de «respecter à tout moment ses parents, de les nourrir, et de les assister en cas de nécessité » 5 .

1. Art. $16, \$ 1$ et 2 . Voir également l'art. $23, \$ 2$ du Pacte international relatif aux droits civils et politiques (PIDCP) du 10 décembre 1966.

2. Art. 16, $\S_{3}$; disposition reprise par les art. 23, $\$ 1 \mathrm{du}$ PIDCP; art. 10 du Pacte international relatif aux droits économiques, sociaux et culturels (PIDESC) du 16 décembre 1966; et art. 18, \$1 de la Charte africaine des droits de l'homme et des peuples (CADHP) du 27 juin 1981. Le deuxième paragraphe de cette disposition de la Charte africaine ajoute que «L'État a l'obligation d'assister la famille dans sa mission de gardienne de la morale et des valeurs traditionnelles reconnues par la Communauté». Le PIDESC ajoute, lui, la nécessité de prendre des mesures spéciales pour protéger les mères (art. 10, \$2) et les enfants et adolescents (art. 10, \$3).

3. Voir également l'art. 7 a) ii) du PIDESC.

4. Voir également l'art. 11 du PIDESC.

5. Art. $29, \S 1$ de la CADHP. Voir également l'art. $27, \$ 1$ de ce même texte. 
Face à ces mentions multiples, les textes européens apparaissent particulièrement peu diserts. Ainsi la Charte des droits fondamentaux de l'Union européenne du 7 décembre 2000 se contente de poser le droit «de se marier et le droit de fonder une famille», qui «sont garantis selon les lois nationales qui en régissent l'exercice» (art. 9), en ajoutant que «la protection de la famille est assurée sur le plan juridique, économique et social» (art. 33). La Convention européenne de sauvegarde des droits de l'homme et des libertés fondamentales du 4 novembre 1950 apparaît elle aussi assez peu concernée par les familles et les droits qui les concernent: au-delà de l'article 12 protégeant le droit de se marier et de fonder une famille pour les «hommes et femmes d'âge nubile», l'article 8 de la Convention, dans une formulation fort synthétique, ajoute que «toute personne a droit au respect de sa vie privée et familiale, de son domicile et de sa correspondance», l'État ne pouvant s'y immiscer que pour des motifs prévus de manière expresse par le deuxième paragraphe de cet article $^{6}$. Postérieurement, l'article 5 du Protocole $\mathrm{n}^{\circ} 7 \mathrm{du}$ 22 novembre 1984 a ajouté que:

[l]es époux jouissent de l'égalité de droits et de responsabilités de caractère civil entre eux et dans leurs relations avec leurs enfants au regard du mariage, durant le mariage et lors de sa dissolution. Le présent article n'empêche pas les États de prendre les mesures nécessaires dans l'intérêt des enfants.

Mais la Convention européenne des droits de l'homme ne peut être lue sans se référer à la jurisprudence de la Cour européenne des droits de l'homme, qui a su donner plein effet aux dispositions de la Convention de 1950. Le contenu largement indéterminé de l'article 8 l'a amenée à effectuer une véritable «construction» du droit au respect de la vie familiale ${ }^{7}$, appliquant ici pleinement la vision selon laquelle il s'agit « d'un instrument vivant à interpréter $[\ldots]$ à la lumière des conditions de vie actuelles ${ }^{8}$. Cette approche est particulièrement visible dans le contentieux dynamique de l'article 8 de la Convention. En effet, la Cour n'a pas hésité à faire preuve en la matière d'un véritable activisme juridictionnel, transformant sa jurisprudence en fonction de l'évolution des mœurs, des conceptions sociales, culturelles et juridiques des États membres du Conseil de l'Europe. L'émergence de «consensus européens » a notamment permis à la Cour de limiter la marge nationale d'appréciation dont jouissent les États dans la mise en œuvre du droit à la vie familiale ${ }^{9}$. En la matière la position de principe de la Cour européenne des droits de l'homme est sans ambiguïté; doivent être pris en compte:

[...] les développements de la société et les changements de perception sociale concernant les questions d'état civil et relationnelles, dont le fait qu'il n'y a pas qu'une seule voie ou un seul choix dans la façon de mener et de vivre sa vie privée et familiale ${ }^{10}$.

La Cour ne s'est donc pas attachée à définir la famille, mais le concept de vie familiale, à la fois plus imprécis et plus large. Suivant une démarche constante depuis la fin des années 1970, les juges de Strasbourg ont cherché à donner du sens à l'article 8 de la Convention, en renforçant peu à peu l'étendue et l'intensité de la protection conférée par cet article. Rendant plus effective la notion de vie familiale (I), ils ont également réussi à expliciter - tout en les encadrant - les droits qui en découlent (II).

\section{L'effectivité de notion de vie familiale}

Utilisant la théorie du «droit vivant», la Cour européenne des droits de l'homme s'est attachée depuis maintenant plus de trente années à définir les contours de la vie familiale, privilégiant en l'espèce une approche concrète (A). Cette construction évolutive, qui a bouleversé nombre d'éléments de droit interne, reste toutefois relativement traditionnelle à l'égard du mariage, qui demeure analysé comme un lien juridique tout à fait spécifique (B).

\section{A. L'appréciation in concreto de la réalité familiale}

La Cour européenne des droits de l'homme n'a jamais donné de définition explicite de la notion de famille, ni de la «vie familiale» envisagée par l'article 8 de la Convention. En ce domaine, la position des juges européens apparaît particulièrement évolutive, afin de « coller» au mieux aux changements sociaux et à l'évolution des mœurs. Comme l'a très justement analysé M. Lévinet, la jurisprudence de Strasbourg

[...] entend moins sauvegarder la famille comme institution que consacrer le droit subjectif de toute personne au respect de ses liens familiaux, de l'intimité de la vie familiale, reconnaître la liberté de chacun de mener la vie familiale de son choix ${ }^{11}$

6. Art. $8, \$ 2$ de la Convention européenne des droits de l'homme: «Il ne peut y avoir ingérence d'une autorité publique dans l'exercice de ce droit que pour autant que cette ingérence est prévue par la loi et qu'elle constitue une mesure qui, dans une société démocratique, est nécessaire à la sécurité nationale, à la sûreté publique, au bien-être économique du pays, à la défense de l'ordre et à la prévention des infractions pénales, à la protection de la santé ou de la morale, ou à la protection des droits et libertés d'autrui».

7. Pour reprendre les mots de F. Sudre, «Rapport introductif», in Le droit au respect de la vie familiale au sens de la Convention européenne des droits de l'homme, F. Sudre (dir.), Bruxelles, Nemesis - Bruylant (Droit et justice; 38), 2002, p. 11.

8. Cour EDH, Tyrer c. Royaume-Uni, 25 avril 1978, \$31.

9. «L'étendue de la marge d'appréciation varie selon les circonstances, les domaines et le contexte; la présence ou l'absence d'un dénominateur commun aux systèmes juridiques des États contractants peut constituer un facteur déterminant à cet égard» (Cour EDH, Rasmussen c. Danemark, 28 novembre 1984, $\$ 40$ ). En d'autres termes, à chaque fois que la Cour constate une communauté de vue préexistante entre les différents États européens, cela réduit leur marge d'appréciation dans la mise en œuvre des droits protégés par la Convention.

10. Cour EDH, Kozak c. Pologne, 2 mars 2010, $\$ 98$.

11. M. Lévinet, «Couple et vie familiale», in Le droit au respect de la vie familiale..., p. 111. 
La famille au sens de la Cour européenne des droits de l'homme apparaît comme une notion à la fois large et souple, qui renvoie à un certain nombre de critères ou d'indices et non à une définition juridique figée. Comme l'avait précisé la Commission européenne des droits de l'homme dès 1986, «la question de l'existence ou non d'une vie familiale est essentiellement une question de fait qui dépend de l'existence réelle et concrète de rapports personnels étroits ${ }^{12}$. Cette analyse in concreto a été reprise par la Cour européenne des droits de l'homme, qui a rappelé à de nombreuses reprises que:

[...] le concept de "vie familiale" visé par l'article 8 ne se borne pas aux seules familles fondées sur le mariage mais peut englober d'autres relations de facto [...]. Pour déterminer si une relation s'analyse en une "vie familiale", il peut se révéler utile de tenir compte d'un certain nombre d'éléments, comme le fait de savoir si les membres du couple vivent ensemble et depuis combien de temps, et s'ils ont eu des enfants ensemble, de manière naturelle ou autre, preuve de leur engagement l'un envers l'autre ${ }^{13}$.

Si la Cour européenne des droits de l'homme n'a jamais été très explicite sur sa grille d'analyse permettant - ou pas - de retenir l'existence d'une vie familiale dans le cadre des différents contentieux dont elle est saisie, l'important volume de la jurisprudence permet aujourd'hui de déceler un certain nombre de critères opérationnels. Apparaissent décisifs l'effectivité des liens interpersonnels qui relient les membres de la famille ${ }^{14}$, mais également l'existence de liens de parenté (voire la simple apparence d'une parenté ${ }^{15}$. Il n'existe donc pas une famille aux yeux de la Cour européenne des droits de l'homme, mais des familles, qui ne renvoient pas toutes aux mêmes situations, projets de vie, ni à la même nature ou intensité de la relation, ni même - évidemment - à une situation juridique identique.

S'il est aujourd'hui impossible de relever l'ensemble des situations de fait qui fondent, selon la Cour, l'existence d'une vie familiale, il reste toutefois possible de dresser les lignes essentielles de cette construction. L'analyse de la jurisprudence permet de découvrir que de nombreux critères, qui pourraient être retenus pour définir la famille, s'avèrent en pratique non décisifs.

Ainsi concernant la situation juridique liant les différents membres de la famille. Avec l'arrêt Marckx c. Belgique du 13 juin 1979, la Cour estime que l'application de l'article 8 concerne tout aussi bien les familles légitimes ${ }^{16}$ que les familles naturelles - et tout spécialement les familles monoparentales -, auxquelles on peut ajouter les concubins ${ }^{17}$. L'existence d'une relation adultérine au regard du droit ${ }^{18}$ ou un mariage polygamique n'empêchent pas davantage la reconnaissance par les juges européens d'une vie familiale entre enfants et parents ${ }^{19}$. Des parents et des enfants nés d'une deuxième union forment également une vie de famille aux yeux de la Cour ${ }^{20}$, tout comme les parents adoptifs et leurs enfants ${ }^{21}$, une famille d'accueil et un enfant ${ }^{22}$, ou le père d'un enfant qu'il n'a pas reconnu ${ }^{23}$. La nationalité des membres de la famille n'est pas plus pertinente pour déterminer ou non une vie familiale ${ }^{24}$. Quant au divorce, il ne met pas fin à la relation familiale unissant parents et enfants ${ }^{25}$.

12. Comm. EDH, K. c. Royaume-Uni, 15 octobre 1986.

13. Cour EDH, X, Y et Z c. Royaume-Uni (GC), 20 mars 1997, \$36. Voir également, inter alia, Cour EDH, Keegan c. Irlande, 26 mai $1994 ;$ Kroon et autres c. Pays-Bas, 27 octobre 1994; Elsholz c. Allemagne (GC), 13 juillet 2000.

14. Dans les arrêts B. c. Allemagne du 28 mai 2009 et T. c. Italie du 13 janvier 2009, les juges européens précisent que «l'existence ou l'absence d'une "vie familiale" au sens de l'article 8 est d'abord une question de fait dépendant de la réalité pratique de liens personnels étroits».

15. Voir, sur ces critères, F. Sudre, "Rapport introductif», p. 20 sq.; F. Sudre, Droit européen et international des droits de l’homme, $11^{\mathrm{e}}$ éd., Paris, PUF, 2012, p. 561 sq.; A. Gouttenoire, "Le droit au respect de la vie familiale», in Les grands arrêts de la Cour européenne des droits de l'homme,

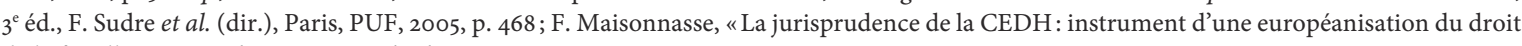
de la famille", Revue des mutations du droit, janvier 2011, p. 5.

16. L'existence d'un mariage véritable (à la fois légal et non fictif) suffit à la Cour pour reconnaître la qualité de la famille à l'ensemble des personnes concernées (voir Cour EDH, Berrehab c. Pays-Bas, 21 juin 1988). Ce qui veut donc dire, a contrario, qu'un mariage blanc ou illégal ne permet pas de reconnaitre ipso facto une famille...

17. Voir Cour EDH, Johnston c. Irlande, 18 décembre 1986; voir également Cour EDH, Şerife Yiğit c. Turquie, 20 janvier 2009: les parents avaient contracté un mariage religieux dont étaient issus six enfants. La Cour, relevant que le couple et leurs enfants mènent une vie commune, conclut à l'existence d'une famille au sens de l'article 8 de la Convention.

18. Cour EDH, Kroon et autres c. Pays-Bas.

19. Comm. EDH, A. et A. c. Pays-Bas, 6 janvier 1992.

20. Cour EDH, X. c. Suisse, 10 juillet 1978.

21. Cour EDH, Jolie et Lebrun c. Belgique, 14 mai 1986; Pini et Bertani, et Manera et Atripadi c. Roumanie, 22 juin 2004. Dans son arrêt Wagner et J. M. W. L. c. Luxembourg du 28 juin 2007 , la Cour a même jugé que les tribunaux nationaux ne peuvent passer outre à un statut juridique d'adoption créé valablement à l'étranger et correspondant à une vie familiale au sens de l'article 8. Il leur est interdit de refuser de reconnaître les liens familiaux qui préexistaient de facto entre la mère adoptive et l'enfant et qui méritent pleine protection. Voir également, Negrepontis et Giannisis c. Grèce, 3 mai 2011.

22. Cour EDH, Moretti et Benedetti c. Italie, 27 avril 2010. Pour la Cour, l'existence de «liens familiaux» entre une famille d'accueil et un enfant doit être prise en compte lors de l'examen d'une demande d'adoption par cette même famille.

23. Cour EDH, Lebbink c. Pays-Bas, $1^{\text {er }}$ juin 2004; Gorgülü c. Allemagne, 26 février 2004.

24. «L'article 8 ne saurait s'interpréter comme comportant pour un État contractant l'obligation générale de respecter le choix, par des couples mariés, de leur domicile commun et d'accepter l'installation de conjoints non nationaux dans le pays» (Cour EDH, Abdulaziz, Cabales et Balkandali c. Royaume-Uni, 28 mai 1985, $\$ 68$ ). La question du droit à la vie familiale des étrangers ne sera pas développée dans cet article. Sur ce point, voir l'article de C.-A. Chassin dans ce numéro, «La vie familiale des étrangers», p. 77.

25. Cour EDH, Berrehab c. Pays-Bas. Dans l'arrêt Chavdarov c. Bulgarie du 21 décembre 2010, la Cour estime qu'une longue cohabitation des deux partenaires et la naissance de trois enfants au cours de celle-ci sont des indices suffisants pour que soit admise l'existence d'une cellule familiale de facto, au sein de laquelle le requérant a pu développer des liens d'affection avec les enfants de sa compagne. 
Le degré de parenté ne constitue pas davantage le facteur décisif d'une vie familiale, puisque celle-ci existe évidemment entre parents et enfants ${ }^{26}$, mais aussi entre frères et sœurs (quel que soit leur âge) ${ }^{27}$, grands-parents et petits-enfants ${ }^{28}$, ou encore oncle (ou tante) et neveu (ou nièce) ${ }^{29}$.

Le sexe ne constitue pas davantage un élément déterminant pour relever l'existence ou non d'une vie familiale, comme le montre l'arrêt $X, Y$ et $Z c$. Royaume-Uni du 22 avril 1997, dans lequel la Cour a indiqué que la relation entre un transsexuel né de sexe féminin, sa compagne et l'enfant de cette dernière (né par insémination artificielle) relevait des «liens familiaux de facto ${ }^{30}$. La parenté est ici remplacée par l'apparence de paternité, le parent transsexuel se comportant en tout point comme le père de l'enfant ${ }^{31}$. Plus récemment, la Cour - en relevant l'évolution existant dans de nombreux États - a admis qu'une relation entre deux personnes de même sexe peut être qualifiée de vie familiale ${ }^{32}$.

On peut enfin relever que la vie familiale ne présuppose pas forcément l'existence d'une cohabitation entre les membres de la famille. Dans ses arrêts Zaunegger $c$. Allemagne du 3 décembre 2009 et Sporer $c$. Autriche du 3 février 2011, la Cour juge ainsi qu'une vie familiale peut exister entre un père et un enfant qui ne vivent pas sous le même toit, à partir du moment où existent un intérêt manifeste et un engagement du père auprès de l'enfant avant et après sa naissance ${ }^{33}$. De même, la privation de liberté d'un individu ne le prive pas de la protection offerte par l'article 8 de la Convention, puisque la Cour a pu reconnaître l'existence d'une vie familiale aux détenus, en soulignant que ceux-ci, même si leur situation entraîne inévitablement des restrictions à l'exercice de ce droit, continuent à bénéficier de l'exercice de la vie familiale ${ }^{34}$.
Par sa jurisprudence, la Cour a opéré une véritable reconstruction de la notion de famille. Mais il reste un point sur lequel les juges de Strasbourg apparaissent plus conservateurs: la prise en compte du lien marital dans les relations familiales.

\section{B. La reconnaissance des effets spécifiques du lien marital}

L'indifférence de la Cour européenne des droits de l'homme à l'égard des liens juridiques unissant les membres de la famille depuis l'arrêt Marckx pourrait laisser penser à une prise en compte très limitée du lien marital dans la recherche des critères fondant une vie familiale. Dans son arrêt Keegan de 1994, la Cour avait ainsi bien confirmé que:

[...] la notion de «famille» visée par cet article [art. 8] ne se borne pas aux seules relations fondées sur le mariage et peut englober d'autres liens «familiaux" de facto lorsque les parties cohabitent en dehors du mariage ${ }^{35}$.

La famille existe donc avant, après et en dehors du mariage, qui ne semble donc plus être le lien déterminant, et qui voit donc son rôle fondateur de la famille «désacralisé » ${ }^{36}$. La Convention européenne des droits de l'homme évoque certes le mariage dans son article 12, mais en tant que droit, appartenant à l'homme et à la femme d'âge nubile ${ }^{37}$. Or, la réalité est plus complexe puisque la Cour continue encore aujourd'hui de reconnaître une spécificité au mariage, qui permet aux époux de se prévaloir de certains droits qui ne sont pas ipso facto reconnus à tous ${ }^{38}$. Les juges européens ont souhaité non seulement protéger la liberté de contracter un mariage,

26. Ainsi, la relation entre une mère et son enfant relève toujours de la vie familiale, quelle que soit la situation matrimoniale de la mère (Cour EDH, Marckx c. Belgique, 13 juin 1979). Ce lien entre parents et enfants apparaît d'ailleurs très logiquement comme la figure la plus évidente de la vie familiale. Pour la Cour, «la notion de famille sur laquelle repose l'article 8 inclut, même en l'absence de cohabitation, le lien entre un individu et son enfant, que ce dernier soit légitime ou naturel. Si ledit lien peut être brisé par des événements ultérieurs, il n'en va ainsi que dans des circonstances exceptionnelles» (Boughanemi c. France, 24 avril 1996, \$35).

27. Cour EDH, Olsson c. Suède, 24 mars 1988; pour des enfants adultes, voir Cour EDH, Boughanemi c. France.

28. Cour EDH, Bronda c. Italie, 9 juin 1988; Pla et Puncernau c. Andorre, 13 juillet 2004.

29. Cour EDH, Boyle c. Royaume-Uni, 28 février 1994.

30. Cour EDH, $X, Y$ et $Z$ c. Royaume-Uni, 22 avril 1997, $\$ 36$.

31. A. Gouttenoire, «La relation parent-enfant dans la jurisprudence de la Cour européenne des droits de l'homme», Informations sociales, $\mathrm{n}^{\circ}$ 149, $2008 / 5$, p. 43.

32. Cour EDH, Schalk et Kopf c. Autriche, 24 juin 2010; la Cour précise «qu'il est artificiel de maintenir le point de vue que, au contraire des couples de sexe opposé, un couple du même sexe ne pourrait pas avoir de "vie de famille" au sens de l'article 8 ». Voir également l'arrêt PB et JS c. Autriche du 22 juillet 2010, dans lequel la Cour évoque l'augmentation du nombre d'États reconnaissant les unions entre personnes du même sexe.

33. Dans l'arrêt Keegan c. Irlande, la Cour a même reconnu l'existence d'une vie familiale entre un père et son enfant... qu'il n'avait alors vu que lors de visites à la maternité. Dans un sens opposé la Cour a pu rejeter l'existence d'une vie familiale pour une mère qui n'est jamais allée voir son bébé à la clinique et s’en est ensuite séparée «avec une indifférence absolue» (Odièvre c. France, 13 février 2003, $\$ 44$ ).

34. Cour EDH, Moisejevs c. Lettonie, 15 juin 2006. Voir F. Sudre, Droit européen..., p. 562 sq.

35. Cour EDH, Keegan c. Irlande, $\$ 44$.

36. Pour reprendre les termes de J.-P. Jacqué, «Le respect de la vie privée dans la jurisprudence de la Convention européenne des droits de l'homme», Annales de l'Université des sciences sociales de Toulouse, t. XXX, 1980, p. 31.

37. Reprenant ainsi - de manière simplifiée - le libellé de l'article $16, \S 1$ de la Déclaration universelle des droits de l'homme, qui prévoit qu' «à partir de l'âge nubile, l'homme et la femme, sans aucune restriction quant à la race, la nationalité ou la religion, ont le droit de se marier et de fonder une famille. Ils ont des droits égaux au regard du mariage, durant le mariage et lors de sa dissolution».

38. À l'image du Code civil français de 1804, dont les textes préparatoires définissent le mariage comme une «convention par laquelle l'homme et la femme s'engagent sous l'autorité de la loi, à vivre ensemble, à nourrir et élever les enfants qui peuvent naître de leur union ». 
mais également l'institution matrimoniale ${ }^{39}$. Dans son arrêt Rees c. Royaume-Uni du 18 octobre 1986, la Cour indique ainsi qu'

[...] en garantissant le droit de se marier, l'article 12 vise le mariage traditionnel entre deux personnes de sexe biologiquement différent [...] le but poursuivi consist[ant] essentiellement à protéger le mariage en tant que fondement de la famille ${ }^{40}$.

Ces références jurisprudentielles pourraient sembler aujourd'hui dépassées, surtout dans un domaine où la Cour européenne a fait preuve d'une particulière propension à faire évoluer sa jurisprudence. Or il n'en est rien, comme le montre l'arrêt Burden c. Royaume-Uni du 29 avril 2008, dans lequel la Grande Chambre rappelle avec force la position traditionnelle de la Cour:

Plutôt que la durée ou le caractère solidaire de la relation, l'élément déterminant est l'existence d'un engagement public, qui va de pair avec un ensemble de droits et d'obligations d'ordre contractuel. [...] il ne peut y avoir d'analogie entre, d'un côté, un couple marié ou en partenariat civil et, de l'autre, un couple hétérosexuel ou homosexuel dont les deux membres ont choisi de vivre ensemble sans devenir des époux ou des partenaires civils $[\ldots]^{41}$.

On peut certes relever que la Cour assimile désormais le lien marital à d'autres «engagements publics», renvoyant ici à d'autres formules qui permettent à deux personnes d'organiser juridiquement leur vie de couple sans pour autant être mariées (à l'image de la loi française n $99-944$ du 15 novembre 1999 instaurant le pacte civil de solidarité - pacs). Mais il n'en reste pas moins que la Cour continue à accepter que les États puissent traiter différemment les couples vivant hors ou dans le cadre d'une relation juridique $^{42}$. Ainsi, dans sa décision Shackell c. Royaume-Uni du 27 avril 2000, la Cour accepte une différence de traitement pour l'octroi de prestations sociales entre, d'une part, une femme célibataire ayant vécu une relation durable avec un homme entre-temps décédé, et, d'autre part, une veuve placée dans la même situation, «car le mariage demeure une institution largement reconnue comme conférant un statut particulier à ceux qui s'y engagent». La Cour a également pu juger conventionnelles les législations étatiques subordonnant l'exigence de l'ouverture d'un droit de pension de survivant à l'existence d'un mariage ${ }^{43}$, ou privant une concubine de longue date de tous droits sociaux et successoraux après le décès de son compagnon ${ }^{44}$. De même la Cour refuse-t-elle de considérer comme ayant des droits identiques un couple marié ou vivant en partenariat, deux sœurs célibataires vivant ensemble ${ }^{45}$, ou bien un homme hébergé par le père d'une amie (que la Cour analyse comme une relation de type économique) ${ }^{46}$.

La question de l'analyse du mariage et de sa portée dans la jurisprudence européenne conduit évidemment à s'interroger également sur la question très actuelle du sexe des époux. En effet, on pourrait penser qu'une Cour rappelant régulièrement son attachement à la «famille traditionnelle» relevant des liens du mariage adopte une lecture particulièrement stricte de l'article 12 de la Convention qui évoque explicitement et exclusivement «l'homme» et «la femme» comme bénéficiaires de cette disposition ${ }^{47}$. Cette position a effectivement été celle de la Commission européenne des droits de l'homme, qui avait estimé en 1989 que la relation d'une requérante avec sa compagne ne donnait "pas lieu à un quelconque droit à se marier et à fonder une famille au sens de l'article 12 de la Convention ${ }^{48}$. Cette position a certes aujourd'hui évolué, même si l'on remarque la persistance d'un certain embarras pour les juges de Strasbourg.

La première évolution émane de l'arrêt Christine Goodwin c. Royaume-Uni du 11 juillet 2002, dans lequel la Cour estime que l'on ne peut plus «aujourd'hui continuer d'admettre que ces termes impliquent que le sexe doive être déterminé selon des critères purement biologiques ${ }^{49}$. Mais cette affaire ne règle pas la question du mariage entre personnes du même sexe, puisque la requérante était une

39. F. Vasseur-Lambry, La famille et la Convention européenne des droits de l'homme, Paris, L'Harmattan (Logiques juridiques), 20oo, p. 159. Voir également M.-T. Meulders-Klein, «Égalité et non-discrimination en droit de la famille. Le rôle des juges", Revue trimestrielle des droits de l'homme, $\mathrm{n}^{\circ}$ 56, octobre 2003 , p. 1194.

40. Cour EDH, Rees c. Royaume-Uni, 18 octobre 1986, $\$ 49$. La Commission européenne avait eu l'occasion de préciser en 1984 son souci de sauvegarder le mariage qui « est dans tous les États signataires de la Convention l'institution reconnue qui entraîne la fondation d'une famille» (déc. $B$., $R$. et J. c. RFA, 15 mars 1984).

41. Cour EDH, Burden c. Royaume-Uni (GC), 29 avril 2008, \$65.

42. Le contrôle de la Cour étant en la matière assez réduit puisqu'elle a eu l'occasion de préciser que, dans ce domaine, les États contractants jouissent d'une ample marge d'appréciation pour déterminer si et dans quelle mesure des différences entre les familles justifient des distinctions de traitement en matière économique ou sociale (Cour EDH, Stec et autres c. Royaume-Uni (GC), 12 avril 2006). Cette position est d'ailleurs partagée par la Cour de Luxembourg qui juge que «la décision de réserver certains avantages aux couples mariés, en excluant tous ceux qui cohabitent sans être mariés, relève soit du choix du législateur, soit de l’interprétation effectuée par les juridictions nationales des règles juridiques de droit interne, sans que les particuliers puissent faire valoir aucune discrimination fondée sur le sexe interdite par le droit communautaire» (CJCE, K. B., 7 janvier 2004, aff. C-117/01, point 28).

43. Cour EDH, déc. Mata Estevez c. Espagne, 10 mai 2001; déc. Manenc c. France, 21 septembre 2010.

44. Cour EDH, Şerife Yiğit c. Turquie, \$29: «en l'absence d’un accord juridique contraignant, il n’est pas déraisonnable que le législateur turc accorde une protection uniquement au mariage civil».

45. Cour EDH, Burden c. Royaume-Uni.

46. Cour EDH, Korelc c. Slovénie, 12 mai 2009.

47. À la différence des termes employés dans l'article 9 de la Charte des droits fondamentaux de l'Union européenne du 7 décembre 2000 , qui évoque de manière plus neutre que "[1]e droit de se marier et le droit de fonder une famille sont garantis selon les lois nationales qui en régissent l'exercice».

48. Comm. EDH, C. M. et L. M. c. Royaume-Uni, 9 octobre 1989.

49. Cour EDH, Christine Goodwin c. Royaume-Uni, 11 juillet 2002, $\$ 100$. 
transsexuelle, passée du sexe masculin au sexe féminin. Il faut attendre l'arrêt Schalk et Kopf c. Autriche du 24 juin 2010 pour que la Cour de Strasbourg prenne position sur ce point, de manière prudente, en se retranchant à nouveau sur la marge nationale d'appréciation reconnue en l'espèce aux États. Selon elle, s'il n'existe toujours pas de «consensus européen » concernant le mariage des couples de même sexe, le droit au mariage consacré à l'article 12 de la Convention ne peut toutefois pas « en toutes circonstances être limité au mariage entre deux personnes de sexe opposés " ${ }^{50}$. Les couples homosexuels peuvent donc mener une vie familiale, mais ceci ne va cependant pas jusqu'à impliquer l'obligation positive pour les États de leur offrir un statut légal, qu'il s'agisse du mariage ou d'un autre statut juridique ${ }^{51}$. La jurisprudence européenne n'est pourtant pas figée sur ce point, puisque la Cour se réserve la possibilité de modifier sa jurisprudence dans ce "domaine évolutif» ${ }^{52}$, afin de rapprocher les statuts juridiques des couples hétérosexuels et homosexuels.

Ce caractère évolutif de la notion de famille et du lien marital caractérise également le contenu des droits dont peuvent se prévaloir tous ceux concernés par la protection offerte par l'article 8 de la Convention. La Cour de Strasbourg a, en effet, opéré dans ce domaine une extension continue - mais encadrée - des droits.

\section{L'extension encadrée des droits liés à l'exercice de la vie familiale}

Depuis maintenant plus de trente ans, la Cour a fait évoluer sa jurisprudence en adoptant une position très ouverte et libérale pour définir la notion de vie familiale. La jurisprudence a également déterminé le contenu des droits qui en découlent, en incorporant à sa jurisprudence des notions externes, qui viennent enrichir les droits protégés par la
Convention et en mobilisant la technique des obligations positives s'imposant aux États ${ }^{53}$. Plus concrètement, la Cour a explicité les droits des couples et des familles en utilisant deux leviers complémentaires, qui sont l'intérêt supérieur de l'enfant (A) et la lutte contre les discriminations (B).

\section{A. La prise en compte de l'intérêt supérieur de l'enfant}

Si la famille renvoie souvent à des liens de parenté et de filiation ${ }^{54}$, toute famille n'est pas forcément composée de parents et d'enfants (ainsi en est-il des deux partenaires de même sexe liés par un pacs, ou d'un couple de concubins sans enfants). La Cour européenne a d'ailleurs eu l'occasion de préciser que si les États doivent permettre aux futurs parents de choisir librement les modalités de naissance de leur enfant ${ }^{55}$, la Convention européenne des droits de l'homme ne protège pas pour autant un quelconque «droit à l'enfant». Les dispositions de l'article 8 de la Convention européenne des droits de l'homme ne garantissent ainsi ni le droit de fonder une famille grâce à une assistance médicale ${ }^{56}$, ni le droit d'adopter ${ }^{57}$.

Par contre, lorsque la cellule familiale comprend des enfants, le maintien de l'unité de cette famille constitue un élément indispensable du respect de l'article 8 aux yeux de la Cour européenne des droits de l'homme. Sa position est aujourd'hui très claire: parmi les éléments de la vie familiale particulièrement mis en exergue figure le droit pour les parents et les enfants d'être ensemble ${ }^{58}$. Afin de rendre ce droit effectif, les États membres ne doivent pas seulement s'abstenir de s'ingérer dans l'exercice de la vie familiale, mais doivent également agir pour protéger l'existence de cette vie familiale, et encore plus précisément pour protéger l'enfant ${ }^{59}$. À ce titre, la notion d' «intérêt supérieur de l'enfant» apparaît comme un véritable fil directeur de la jurisprudence européenne, permettant d'expliciter

50. Cour EDH, Schalk et Kopf c. Autriche, 24 juin 2010, $\$ 61$.

51. Voir A. Gouttenoire, «La famille dans la jurisprudence de la Cour européenne des droits de l’homme», Droit de la famille, avril 2011 , étude n 10 , $\$ 7$.

52. Cour EDH, Schalk et Kopf c. Autriche, $\$ 105$.

53. Cour EDH, Marckx c. Belgique. Dans son arrêt Airey c. Irlande du 9 octobre 1979, la Cour avait également précisé que si l'article 8 a «essentiellement pour objet de prémunir l'individu contre les ingérences arbitraires des pouvoirs publics, il ne se contente pas de demander à l'État de s'abstenir de pareilles ingérences : à cet engagement plutôt négatif peuvent s'ajouter des obligations positives inhérentes à un respect effectif de la vie privée ou familiale» $(\$ 32)$. Ceci permet de «protéger des droits non pas théoriques ou illusoires, mais concrets et effectifs» (\$24). Voir également Cour EDH, Ignaccolo-Zenide c. Roumanie, 25 janvier 2000 ; Bianchi c. Suisse, 22 juin 2006.

54. Voir A. Batteur, Droit des personnes, des familles et des majeurs protégés, $6^{\mathrm{e}}$ éd., Paris, LGDJ-Lextenso, 2012, p. 13 sq.

55. Voir Cour EDH, Ternovsky c. Hongrie, 14 décembre 2010, concernant le choix d'un accouchement à domicile.

56. Dans son arrêt $S$. H. et autres $c$. Autriche du $1^{\mathrm{er}}$ avril 2010, la Cour rappelle ainsi (conformément à sa jurisprudence antérieure issue notamment des arrêts Evans c. Royaume-Uni et Dickson c. Royaume-Uni des 10 avril et 4 décembre 2007) que l'utilisation de la procréation médicalement assistée constitue certes un droit protégé par l'article 8 de la Convention européenne des droits de l'homme, mais que l'absence d'approche européenne uniforme de cette technique ne contraint pas les États à l'autoriser. L'arrêt S. H. et autres c. Autriche (GC) du 3 novembre 2011 ajoute qu'en l'absence d' «une claire communauté de vues entre les États membres» ( $\$ 69)$, les juges de Strasbourg renvoient au législateur national le soin de choisir quelles seront les techniques de procréation médicalement assistées licites ou non (sous réserve de justifier l'interdiction si l'État fait ce choix). La Cour observe toutefois que ce domaine de la procréation artificielle connaît des évolutions scientifiques et juridiques particulièrement rapides, et appelle un examen permanent de la part des États membres.

57. Cour EDH, E. B. c. France, 22 janvier 2008.

58. Cette analyse étant partagée par la Convention du Conseil de l'Europe sur les relations personnelles concernant les enfants du 15 mai 2003 , qui

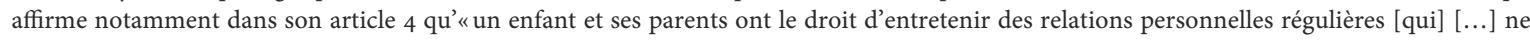
peuvent être restreintes ou exclues que lorsque cela est nécessaire dans l'intérêt supérieur de l'enfant».

59. Voir Cour EDH, Elsholz c. Allemagne, $\$ 52:$ : Il y a lieu de faire prévaloir l'intérêt des enfants lorsque sont en jeu les droits garantis aux parents par l'article 8 de la Convention et ceux des enfants». 
nombre d'obligations incombant aux États membres ${ }^{60}$, qui se déploient aujourd'hui dans deux domaines essentiels.

En premier lieu, la Cour a encadré les différentes situations dans lesquelles un enfant peut être séparé de ses parents ${ }^{61}$ afin de le protéger, en réduisant progressivement la marge d'appréciation dont bénéficient les États en l'espèce ${ }^{62}$. Les droits des parents peuvent alors subir de fortes restrictions, à partir du moment où les mesures de protection sont temporaires ${ }^{63}$, justifiées et proportionnées ${ }^{64}$, et qu'elles permettent de maintenir une relation entre l'enfant et ses parents ${ }^{65}$. En effet, le respect de l'intérêt supérieur de l'enfant nécessite que sa prise en charge par les autorités publiques ne mette pas fin aux relations familiales naturelles ${ }^{66}$.

En second lieu, de nombreux arrêts visent à protéger les droits de l'enfant à la suite d'une séparation parentale, ou d'un divorce. Ni la Convention, ni la jurisprudence de la Cour n'interdisent évidemment de telles ruptures. Mais les juges de Strasbourg essaient dans de telles circonstances de protéger au maximum les enfants et de maintenir les liens avec leurs parents, "même lorsque la relation entre les parents s'est rompue ${ }^{67}$. La Cour a notamment été amenée à rappeler que l'article 8 inclut le droit pour le parent divorcé non investi du droit de garde d'avoir des contacts avec son enfant et de lui rendre visite, ce qui passe par des mesures prises par l'État pour rendre ce droit de visite effectif ${ }^{68}$, qui peuvent même aller jusqu'à des sanctions à l'égard du parent avec qui vit l'enfant et qui ne respecte pas les droits de l'autre parent ${ }^{69}$. À ce titre, la Cour est particulièrement exigeante à l'égard des États dans les hypothèses d'enlèvement d'enfant. L'arrêt Ignacollo-Zenide c. Roumanie de 2000 rappelle en effet qu'il leur incombe de prendre toutes les mesures nécessaires au retour de l'enfant ayant fait l'objet d'un déplacement illicite, conformément aux dispositions de la Convention de La Haye du 25 octobre 1980 sur les aspects civils de l'enlèvement international d'enfants ${ }^{70}$. Mais il ne peut jamais s'agir d'un retour automatique de l'enfant, qui doit voir respecté son développement personnel, en prenant en compte « son âge, sa maturité, la présence ou l'absence de ses parents, l'environnement dans lequel il vit et son histoire personnelle ${ }^{71}$. Concernant l'ensemble de ces situations de garde d'enfant suite à divorce ou séparation, la Cour apparaît particulièrement attentive à la rapidité d'action des autorités étatiques, ainsi qu'à l'effectivité des mesures qui permettent de mettre en œuvre les décisions rendues par les tribunaux ${ }^{72}$.

À cette utilisation continue de la notion d' «intérêt supérieur de l'enfant», s'est ajoutée une active politique jurisprudentielle de lutte contre les discriminations, qui a également permis d'étendre les droits relevant de la vie familiale.

\section{B. La lutte contre les discriminations frappant les membres de la famille}

La lutte contre les discriminations pouvant frapper les membres de la famille constitue un point important d'une jurisprudence européenne cherchant à progressivement effacer les différences de traitement non justifiées frappant les enfants, mais aussi les parents, et même les partenaires des couples. Ces jurisprudences associant les articles 8 et $14^{73}$ de la Convention européenne des droits de l'homme

60. Voir Cour EDH, Wagner et J. M. W. L. c. Luxembourg; Maire c. Portugal, 26 juin 2003. Cette notion d'«intérêt supérieur de l'enfant» est prévue par l'article $3 \$ 1$ de la Convention internationale relative aux droits de l'enfant du 20 novembre 1989, selon lequel «Dans toutes les décisions qui concernent les enfants, qu'elles soient le fait des institutions publiques ou privées de protection sociale, des tribunaux, des autorités administratives ou des organes législatifs, l'intérêt supérieur de l'enfant doit être une considération primordiale». Cette disposition constitue pour la Cour de Strasbourg une norme de référence qu'elle mobilise régulièrement afin de renforcer ses solutions jurisprudentielles.

61. La Cour impose même aux autorités étatiques de retirer l'enfant de son milieu familial dans certaines circonstances particulièrement graves. Tout défaut d'action de l'État sera alors constitutif d'une violation non seulement de l'article 8 de la Convention, mais également de son article 3 . Ainsi, dans l'arrêt Z. et autres c. Royaume-Uni du 10 mai 2001, les juges de Strasbourg condamnent l'État pour ne pas avoir pris, pendant plus de quatre ans, les mesures nécessaires pour protéger des enfants contre les actes de maltraitance qu'ils subissaient au domicile familial. La Cour a, en effet, eu l'occasion de préciser que «l'article 8 ne saurait en aucune manière autoriser un parent à prendre des mesures préjudiciables à la santé et au développement de son enfant» (Cour EDH, Gnahoré c. France, 19 septembre 2000, \$51).

62. Même si la Cour continue à n'exercer qu'un contrôle restreint sur la mesure même de placement, en reconnaissant aux États une très large marge d'appréciation. Voir Cour EDH, K. A. c. Finlande, 14 janvier 2003.

63. Cour EDH, Olsson c. Suède ( $\left.n^{\circ} 1\right), 24$ mars 1988. Pour la Cour, la fin d'une prise en charge d'un enfant par les services sociaux s'impose «si l'amélioration des circonstances l'ayant entraînée semble probablement consolidée». Par contre, «ne restituer un enfant à ses parents que pour le reprendre en charge peu après desservirait à coup sûr ses intérêts» (\$76). Voir également Cour EDH, Amanalachioai c. Roumanie, 26 mai 2009.

64. Cour EDH, Johansen c. Norvège, 7 août 1996; V. c. France, 26 novembre 2009

65. Cour EDH, Gnahoré c. France; Kutzner c. Allemagne, 26 février 2002.

66. Cour EDH, Vautier c. France, 26 novembre 2009.

67. Cour EDH, Eriksson c. Suède, 22 juin 1989, \$22. Voir également, inter alia, Keegan c. Irlande; Hoffmann c. Allemagne, 11 janvier 2002.

68. Cour EDH, Olsson c. Suède; Fourchon c. France, 28 juin 2005; Bove c. Italie, 30 juin 2005; Reigado Ramos c. Portugal, 22 novembre 2005 ; Gluhakovic c. Croatie, 12 avril 2011.

69. Cour EDH, Cengiz-Kiliç c. Turquie, 6 décembre 2011.

70. Voir également, inter alia, Cour EDH, Bianchi c. Suisse; Mamousseau et Washington c. France, 6 décembre 2007; L. et F. c. Belgique, 17 juillet 2008.

71. Cour EDH, Neulinger et Shuruk c. Suisse (GC), 6 juillet 2010, $\$ 138$.

72. Pour la Cour, «le passage du temps» peut en effet avoir des conséquences irrémédiables sur les relations entre l'enfant et le parent donc il est séparé (Cour EDH, Maire c. Portugal, $\$ 74$ ). Sur ces points, voir N. Mathieu, «Séparation des parents et garde d'enfant - le point sur la jurisprudence de la Cour européenne des droits de l'homme», Revue trimestrielle des droits de l'homme, $\mathrm{n}^{\circ}$ 93, janvier 2013 , p. 50 sq.

73. «La jouissance des droits et libertés reconnus dans la présente Convention doit être assurée, sans distinction aucune, fondée notamment sur le sexe, la race, la couleur, la langue, la religion, les opinions politiques ou toutes autres opinions, l'origine nationale ou sociale, l'appartenance à une minorité nationale, la fortune, la naissance ou toute autre situation ». 
se sont développées en deux volets complémentaires, luttant contre les discriminations liées au statut juridique des membres de la famille et condamnant celles liées au sexe, ce qui a notamment permis de mieux faire respecter le droit à la vie familiale des homosexuel(le)s.

L'arrêt Marckx constitue la première étape de cette lutte contre les discriminations familiales. Face à une requérante qui se plaignait que le droit belge restreignait ses possibilités de léguer ses biens à sa fille en raison de son statut de mère célibataire, la Cour a analysé une telle situation comme une discrimination injustifiée ne garantissant pas à l'enfant les droits dont pouvaient se prévaloir les enfants des familles légitimes. Cette solution a été postérieurement étendue par l'arrêt Vermeire c. Belgique du 29 novembre 1991 aux discriminations frappant les enfants légitimes dans la succession des grands-parents. Aux mêmes périodes, la Cour a également jugé inconventionnelle la loi autrichienne qui attribuait la préséance aux enfants nés dans le cadre du mariage au détriment des enfants nés en dehors du mariage, pour ce qui concerne l'attribution des terres agricoles par voie de succession $a b$ intestat $^{74}$. Cette ligne jurisprudentielle n'a pas été démentie par la suite, comme le démontre le célèbre arrêt Mazurek $c$. France du $1^{\mathrm{er}}$ février 2000, qui a sanctionné la législation française qui limitait alors les droits successoraux des enfants adultérins par rapport à ceux des enfants légitimes, ou lorsque la Cour a refusé que les juges nationaux réservent la transmission des successions aux seuls enfants ou petits-enfants nés d'un "mariage légitime et canonique ${ }^{75}$. Confirmant cette solution, le récent arrêt Fabris c. France du 7 février 2013 a permis à la Grande Chambre de la Cour de synthétiser sa jurisprudence sur tous ces points, en rappelant qu'

[e]lle n'a eu de cesse de réaffirmer [1]e principe fondamental érigeant l'interdiction de discrimination fondée sur le caractère «naturel» du lien de parenté en norme de protection de l'ordre public européen ${ }^{76}$.

Constatant en l'espèce l'existence d'un consensus européen en la matière, elle relève

[...] l'importance de l'égalité de traitement entre enfants issus du mariage et enfants nés hors mariage [...], ce qui a d'ailleurs conduit aujourd'hui à l'uniformité des législations nationales en la matière [...] ainsi qu'à une évolution sociale et juridique qui entérine définitivement l'objectif d'égalité entre les enfants ${ }^{77}$.

$\mathrm{Au}$-delà des questions spécifiques de successions, la lutte contre les discriminations en raison du statut juridique des père et mère a également concerné les questions de parentalité. Ainsi, le refus pour une mère célibataire de se voir reconnaître, au Luxembourg, les liens familiaux créés par un jugement étranger, viole les articles 8 et 14 de la Convention ${ }^{78}$. De même que l'impossibilité pour les pères non mariés et séparés de leur ancienne compagne de déduire du revenu imposable la pension alimentaire versée à la mère pour subvenir aux besoins de l'enfant qu'ils ont eu ensemble ${ }^{79}$, que le traitement différencié pour des pères divorcés et des pères d'enfants nés hors mariage en matière de droit de visite après une séparation ${ }^{80}$, ou que l'impossibilité pour un père non marié de participer à l'exercice de l'autorité parentale sans l'accord de la mère ${ }^{81}$.

Combattre les discriminations liées au sexe a constitué le deuxième axe jurisprudentiel de la Cour. Dès l'arrêt Abdulaziz, Cabales et Balkandali de 1985, la Cour rappelle que «la progression de l'égalité entre les sexes constitue [...] un objectif important des États membres ${ }^{82}$. Appliquant ce raisonnement, la Cour de Strasbourg a ainsi pu juger que la législation turque obligeant les femmes à porter le nom de leur mari après le mariage était constitutive d'une discrimination fondée sur le sexe, le droit national n'imposant pas aux maris de modifier leur nom patronymique ${ }^{83}$. Le récent arrêt Konstantin Markin c. Russie (GC) du 22 mars 2012 constitue un autre exemple caractéristique d'application de cet objectif. Dans cette affaire qui concernait un militaire russe qui s'était vu refuser par les autorités un congé parental pour s'occuper de ses trois enfants après son divorce, la Cour condamne sans surprise la Russie pour violation des articles 14 et 8 . Dans sa démonstration, la Cour rappelle que dans des "sociétés européennes contemporaines [qui] ont évolué vers un partage plus égalitaire entre les hommes et les femmes des responsabilités en matière d'éducation des enfants ${ }^{84}$, il est impossible de maintenir de telles législations ayant «pour effet de perpétuer les stéréotypes liés au sexe» qui

74. Cour EDH, Inze c. Autriche, 28 octobre 1987.

75. Cour EDH, Pla et Puncernau c. Andorre.

76. Cour EDH, Fabris c. France (GC), 7 février 2013, $\$ 57$.

77. Ibid., $\$ 58$.

78. Cour EDH, Wagner et J. M. W. L. c. Luxembourg.

79. Cour EDH, M. c. Royaume-Uni, 19 juillet 2005.

80. Cour EDH, Elsholz c. Allemagne; Sommerfield et Sahin c. Allemagne, 8 juillet 2003.

81. Cour EDH, Zaunegger c. Allemagne, 3 décembre 2009. Dans son arrêt Sporer c. Autriche du 3 février 2011, la Cour ajoute que s'il n'existe aucun consensus européen sur la question de savoir si le père d'un enfant naturel a le droit de demander le partage de l'autorité parentale contre la volonté de la mère, la majorité des États membres semble considérer que l'attribution de cette prérogative doit reposer sur l'intérêt supérieur de l'enfant et qu'elle doit être soumise au contrôle des juridictions internes en cas de conflit entre les parents.

82. Cour EDH, Abdulaziz, Cabales et Balkandali c. Royaume-Uni, $\$ 78$. Les requérantes contestaient la loi anglaise de l'époque qui permettait plus aisément à un homme installé au Royaume-Uni qu'à une femme dans la même situation d'obtenir, pour son conjoint non national, l'autorisation d'entrer ou de rester dans le pays.

83. Cour EDH, Ünal Tekeli c. Turquie, 16 novembre 2004. Voir également Burghartz c. Suisse, 22 février 1994.

84. Cour EDH, Konstantin Markin c. Russie (GC), 22 mars 2012, §139. 
constituent "un désavantage tant pour la carrière des femmes que pour la vie familiale des hommes ${ }^{85}$.

Cette lutte contre les stéréotypes sexistes a également permis de faire évoluer la perception des couples et des familles homosexuels, en les «banalisant» et en alignant leurs droits sur ceux de leurs homologues hétérosexuels ${ }^{86}$. Dès 1999, la Cour refuse ainsi que l'autorité parentale ne soit pas accordée en raison de l'orientation sexuelle d'un parent ${ }^{87}$ et l'arrêt E. B. c. France du 22 janvier 2008 juge qu'un rejet de demande d'agrément en vue d'adopter ne peut être fondé sur la seule orientation sexuelle du demandeur. C'est dans cette ligne très libérale que s'inscrit l'arrêt X. et autres c. Autriche (GC) du 7 mars 2013, qui juge que l'exclusion non justifiée des couples homosexuels des procédures d'adoption constitue une discrimination contraire à la Convention. Certes, cette décision n'oblige pas explicitement les États à ouvrir l'adoption aux couples homosexuels. On peut toutefois penser que la solution adoptée par la Cour ne permet plus aux États de refuser l'adoption coparentale aux couples homosexuels mariés à partir du moment où les autres couples mariés y ont accès.

Comme dans d'autres domaines, l'œuvre jurisprudentielle de la Cour européenne des droits de l'homme relative à la famille et aux droits de ses membres peut amener à des analyses très divergentes. Certains regretteront probablement l' "explosion" de la notion de famille traditionnelle, d'autres déploreront des avancées trop timides concernant le mariage ou les droits parentaux reconnus aux homosexuels. La Cour est elle-même consciente de la complexité de sa tâche, qu'elle qualifie d' «exercice par nature difficile et délicat», puisqu'il consiste en la «recherche d'un équilibre entre la préservation de la famille traditionnelle» et d'autres droits (dont ceux des minorités sexuelles, mais aussi ceux des femmes, des enfants, des pères, des mères...), ce qui «peut obliger les États à concilier des vues et des intérêts concurrents perçus par les parties concernées comme étant fondamentalement antagonistes ${ }^{88}$. L'interprétation évolutive de la Convention européenne des droits de l'homme ${ }^{89}$, qui consiste à accompagner les transformations et non à les précéder ni à les imposer de force aux États membres, relève d'une politique jurisprudentielle prêtant par nature le flanc aux critiques ${ }^{90}$. La jurisprudence de la Cour a au moins permis d'imposer l'idée qu'il n'existe pas de famille «standard» ou «traditionnelle», dont la composition doit être prédéterminée par le droit. Adoptant une approche pluraliste, elle bouleverse l'analyse civiliste selon laquelle le droit de la famille renvoie seulement à l'alliance et à la filiation. Elle œuvre enfin de manière indispensable à la lutte contre les discriminations frappant les membres des familles. Mais en la matière les solutions apportées tant par les juges que par les législateurs risquent d'être toujours en relatif décalage avec les évolutions sociales, car comme l'avait fort justement relevé le doyen Carbonnier, «le droit n'intervient qu'avec un temps de retard légitime $»^{91}$.

85. Ibid., $\$ 141$. La Cour n’exige toutefois pas une égalité parfaite des hommes et femmes dans l'exercice des droits familiaux. Ainsi, l'institution de délais pour l'engagement d'une action en désaveu de paternité peut être justifiée car elle empêche les pères d'abuser du droit de contester leur paternité en exerçant tardivement l'action en question. Ce délai poursuit l'objectif légitime de garantir aux enfants la sécurité juridique quant à leur statut (Cour EDH, Rasmussen c. Danemark). De même pour le maintien d'un dispositif législatif qui favorise le départ à la retraite des femmes en prenant en compte le nombre d'enfants élevés. Ce traitement discriminatoire est jugé par la Cour «raisonnablement et objectivement» justifié (Cour EDH, Andrle c. République tchèque, 17 février 2011, \$60).

86. Et ce dans tous les domaines comme le montre l'arrêt Kozak c. Pologne dans lequel la Cour prohibe le refus de transmission du bail à un homme qui vivait depuis plusieurs années en concubinage avec le locataire de l'appartement (le droit polonais ne reconnaissant que le concubinage hétérosexuel). Sur ces questions, voir L. Mauger-Vielpeau, J.-M. Larralde, "Les interdits relatifs à la famille homosexuelle", in Les grandes décisions du droit des personnes et de la famille, A. Batteur (dir.), Paris, LGDJ-Lextenso, 2012, p. 222 sq.

87. Cour EDH, Salgueiro Da Silva Mouta c. Portugal, 21 décembre 1999.

88. Cour EDH, X. et autres c. Autriche, 19 février 2013, \$151. Voir également Kozak c. Pologne, $\$ 99$.

89. Voir C. Rozakis, "The Particular Role of the Strasbourg Case-Law in the Development of Human Rights in Europe», Nomiko Vima, numéro spécial, 2010, European Court of Human Rights 50 Years, p. 25 sq.

90. Voir à ce titre l'opinion partiellement dissidente commune aux juges Casadevall, Ziemele, Kovler, Jočienè, Šikuta, de Gaetano et Sicilianos sous l'arrêt $X$. et autres $c$. Autriche.

91. J. Carbonnier, Droit civil, t. 2, La famille, l'enfant, le couple, $20^{\mathrm{e}}$ éd., Paris, PUF (Thémis. Droit privé), 1999, p. 7. 